\title{
Gender Based Violence and Health Effects
}

\section{Tazeen Saeed Ali}

Associate Professor, Assistant Dean of Research and Graduate studies, School of Nursing and Midwifery, Department of Community Health Sciences, Aga Khan University, Pakistan. Email: tazeen.ali@aku.edu, ORCiD: 0000-0002-8896-8766

Gender Based Violence (GBV) violates the basic human right of a person's physical, sexual and emotional integrity and health such that globally, one out of every three women is subject to some form of violence, ranging from verbal and physical abuse to emotional blackmailing and psychological torture eventually leading to futile despair and hopelessness. The ironical perception of gender equality in our society is the root cause of gender-based violence, with women being more afflicted by such a form of gender discrimination than men.'

GBV can occur at all levels regardless of religions, cultures, languages and communities, thus depriving women of their right to speak up for themselves. ${ }^{2}$ Among all forms (Physical, Sexual, psychological) of violence physical abuse, is of a severe form, is now outrageously considered as acceptable by the typical Pakistani male dominant society, which believes in their dominance and treat women as mere puppets needed to appease sexual desires of the men. The risk of being subjected to domestic violence is also elevated if the husband is addicted to drugs and alcohol. ${ }^{2}$ However, in all circumstances, in most Muslim countries like Pakistan, a woman's disobedience of societal and gender norms is equated to disobedience of her husband simply because she wishes to speak up for her rights. Unfortunately, the home in which the girl is born is also where her voice is first suppressed, and hence she learns that everywhere else in life, whether it be the work place, or later after getting married, she is supposed to crush her dreams, shatter her goals, compromise independently in the face of adversity, do all the household work $24 / 7$ often without getting rest or respect.

These different forms of violence affect women; leading them to contract different illness including systemic and variety of psychiatric. The commonly observed physical symptoms are shoulder aches, backaches, and at times overeating due to depression leaving them obese further aggravating the situation including diabetic mellitus, arthritis etc. Moreover, injuries and trauma are most common consequent of physical violence. The developing mental illnesses tend to leave women with suicidal thoughts and tendencies, and potentially often result to suicide attempts, some of which succeed as well. ${ }^{3}$ The other symptoms including lack of focus and problems in performing daily routine activities, loss or hindered ability of decision making, loss of interest in previously enjoyable things, loss of self-esteem and feelings of worthlessness, often leading to major depression, ${ }^{4}$ A review article from Pakistan in which a number of studies were analyzed found that the prevalence of anxiety and depression ranged between 25 and $66 \%$ among married women. ${ }^{5}$

The woman may also be otherwise driven to an extent that she starts considering such an abuse to be 'normal' and mild or acceptable, resulting in developing a serious lack of self-esteem and self-confidence as evident via studies in Sweden. ${ }^{6}$ In fact, studies made in Palestine and Ethiopia have made it vivid that the risk of lack of self-esteem is proportional to the period of existence of an abusive relationship. ${ }^{6}$ While all of this is happening to the woman, the girl's family often isolates itself from all responsibility of helping the girl in this difficult situation. ${ }^{6}$

One of the most prominent underlying causes of GBV is the relatively higher rate of illiteracy amongst women who are ignored either by cultural norms or family beliefs, both of which generally prioritize males over females and hence prefer educating the son rather than the daughter.' The main element behind this drop that literacy is directly empowers woman and elevating her status particularly by making her more aware of her rights and financially independent which ultimately develops insecurities in the typical Pakistani male dominant society by underrating his much inflated ego, ultimately leading to conflicts and eventually violence. ${ }^{2}$ According to a Sudanic study while also proving why unemployed and uneducated women are much more likely to get abused. ${ }^{2}$ On the other hand, neither should the female always be held responsible for infertility nor should she ever be blamed for not given birth to a male 
offspring because it is in fact the male who should be held accountable because it is he who provides the $X$ and $Y$ chromosomes. However, our society is either unaware or has conveniently decided to overlook this pertinent fact, which is the very factor that becomes the cause of fights, violence and at times divorce; with associated depression and ignorance for women, especially those who give birth to a daughter. ${ }^{1}$ If a woman, on the other hand, is infertile, she often has to face severe consequences which may include the husband remarrying, abusing or divorcing her etc.; all of which lead to stress, sorrow, shame and lowered selfesteem for the woman, as proven by studies conducted in India and Bangladesh together with those made in India and Thailand. ${ }^{\text {? }}$

In order to eradicate such institutionalized concepts of gender inequality, there is a definite need for an increase in the number of active legal institutions that women can rely upon. ${ }^{1}$ This is essential because in Pakistan, many abused women do not want to get divorced while those who do want to are either not able to find any legal assistance due to the particulate discrimination of the law against women or are not financially strong enough to seek such assistance.' Therefore, certain measures must be taken to curb this critical issue of gender based violence. Firstly, there is a need to recognize the problem, and for the government to actively resolve this issue at a national level by investing efforts such as authorizing commissions and allocating budgets which would as a result help recognize, authorize and empower women by guaranteeing them an adequate level of safety and security while also ensuring them the provision of equal opportunities of getting employed. Secondly, the government should also initiate proper screening of all types of domestic violence and abuse in healthcare settings while adequate emergency treatments with rehabilitative measures should also be provided. Apart from this, it is also necessary to make the study of GBV a must part of the curricula of nursing and medical schools in Pakistan. At the same time, it is crucial to conduct awareness programs for both women and men alike, by using valuable resources like trained nurses, doctors and psychologists whereas the further associated necessity of redefining the rights of women in the light of Islamic teachings and literature may be fulfilled by assigning this responsibility to a committee comprising of religious leaders, Ulema, and scholars. While there is definitely a need for must change in the police department and judicial processes, women police should also be trained to deal with women facing domestic violence in order to make women feel more secure and protected. Moreover, results of various studies demonstrate an alarming prevalence of genderbased violence against females as well. The governing problems of criminalization, homelessness, and poor availability of management are the major factors correlating with GBV against females. Socio-legal policy reforms, improved access to housing and drug treatment, and scale up of violence prevention efforts, including awareness and educational programs will definitely prove crucial to stem $\mathrm{GBV}{ }^{2-4}$

Summarizing in the end, to ensure strengthened social, political and economic status of women, the elimination of violence against women will require a must change in our patriarchal system which would consequently lead to her empowerment as an equal human being. In addition due to repeated violence number of morbidities increase thereby further increasing burden on health system of Pakistan. Conclusively, violence prevention programs utilizing coaches as positive messengers for respect and nonviolence can also serve as a useful addition to globally prevent and reduce violence against women all over the world.

\section{REFERENCES}

1. Ali TS, Krantz G, Gul R, Asad N, Johansson E, Mogren I. Gender roles and their influence on life prospects for women in urban Karachi, Pakoistan: a qualitative study. Global Health Action 2011; 4:7448.

2. Ali TS, Bustamante Gavino I. Prevalence of and reasons for domestic violence among women from low socioeconomic communities of Karachi. 2007.

3. Deyessa N, Berhane Y, Alem A, Hogberg U, Kullgren G. Depression among women in rural Ethiopia as related to socioeconomic factors: A community-based study on women in reproductive age groups. Scand J Public Health 2008; 36:589-97.

4. Hammar A, Ardal G. Cognitive functioning in major depression a summary. Front Hu Neurosci 2009; 3:1-7.

5. Mirza I, Jenkins R. Risk factors, prevalence, and treatment of anxiety and depressive disorders in Pakistan: systematic review. Br Med J 2004; 328:794-7.

6. Ali T S, Mogren I, Krantz G. Intimate partner violence and mental health effects: A population-based study among married women in Karachi, Pakistan. Int J Behav Med 2013; 20:131-9.

7. Sami N, Ali TS. Psycho-social consequences of secondary infertility in Karachi. J Pak Med Assoc 2006; 56:19. 